\title{
Propriedades antimicrobianas do kefir
}

\section{Antimicrobial properties of kefir}

\author{
Priscila Alves Dias ${ }^{1 *}$, Janaína Viana da Rosa', Talita Scheneid Tejada', Cláudio Dias Timm¹
}

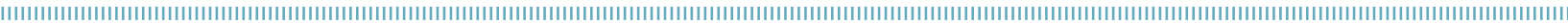

RESUMO: Os leites fermentados têm sido amplamente consumidos desde a Antiguidade e, atualmente, comercializados em todo o mundo. Kefir é um leite fermentado, ácido, levemente alcoólico, produzido artesanalmente a partir da fermentaçáo do leite pelos grãos de kefir, os quais contêm uma população estável de micro-organismos. O processo fermentativo gera uma série de compostos que conferem sabor e aroma característicos ao kefir, além de substâncias bioativas, responsáveis por propriedades nutracêuticas. A utilizaçáo de micro-organismos com propriedades antimicrobianas como conservantes naturais é uma alternativa que tem como vantagem a inibição do desenvolvimento de bactérias deteriorantes e patogênicas sem o uso de substâncias químicas indesejáveis. O interesse em tais espécies aumentou nos últimos anos, viabilizando o uso desses micro-organismos probióticos como conservantes e inibidores naturais em vários produtos de origem animal, como leites, carnes e derivados. O objetivo deste trabalho foi apresentar uma revisão da literatura sobre as propriedades antimicrobianas atribuídas ao kefir.

PALAVRAS-CHAVE: kefir; atividade antimicrobiana; substâncias bioativas.

\begin{abstract}
The fermented milks have been consumed since the antiquity, and currently it have been widely marketed and consumed around the world. Kefir is a fermented, acid, mildly alcoholic homemade milk produced from the fermentation of milk by kefir grains which contain a stable population of microorganisms. The fermentation process generates a series of compounds which impart flavor and aroma to kefir, and bioactive substances responsible for nutraceutic properties. The use of micro-organisms with antimicrobial properties as natural preservative is an alternative that has the advantage of inhibiting the development of pathogenic and spoilage bacteria without the use of undesirable chemicals. The interest in these species has increased in recent years, enabling the use of these probiotic micro-organisms as preservatives and natural inhibitors in several animal products such as milk, meat and their products. The aim of this paper is to present a review of literature on the antimicrobial properties attributed to kefir.
\end{abstract}

KEYWORDS: kefir; antimicrobial activity; bioactive substance. 
A origem dos leites fermentados remete à Antiguidade, provavelmente ao momento em que o homem começou a utilizar o leite dos animais na sua alimentaçâo. As tribos nômades adquiriram o hábito de conservar o leite em odres e recipientes de cerâmica ou peles de animais, onde o leite acabava fermentando. Logo, observavam um produto cuja vida útil era mais prolongada que a da matéria-prima. Desse modo, desenvolveu-se uma tecnologia empírica e foram surgindo os diferentes tipos de leites fermentados que, desde então, têm sido amplamente consumidos e comercializados em todo o mundo (OrDóŃEZ, 2005).

Leite fermentado é o produto resultante da fermentação do leite por fermentos lácteos próprios, segundo o Regulamento Técnico de Identidade e Qualidade de Leites Fermentados do Ministério da Agricultura, Pecuária e Abastecimento. Esse tipo de alimento inclui produtos como iogurte, leite cultivado ou fermentado, leite acidófilo, kefir, kumys e coalhada (Brasil, 2000). Kefiré um leite fermentado, ácido, levemente alcoólico, produzido artesanalmente a partir de grãos que contêm uma população relativamente estável de micro-organismos (Abraham; De Antoni, 1999). O processo fermentativo gera uma série de compostos que conferem sabor e aroma característicos ao kefir, além de substâncias bioativas, responsáveis por propriedades nutracêuticas (AHMED et al., 2013).

Estudos têm demonstrado que o consumo regular de kefir traz uma série de benefícios à saúde, tais como estimulaçáo do sistema imune (VINDERola et al., 2005), atividade antimicrobiana contra patógenos (Rodrigues et al., 2005), equilíbrio da microbiota intestinal (MARQuina et al., 2002) e açấo antitumoral (FARNwORTH et al., 2005). A utilização de micro-organismos com propriedades antimicrobianas como conservantes naturais é uma alternativa que tem como vantagem a inibição do desenvolvimento de bactérias indesejáveis sem o uso de substâncias químicas (Pelaez, 2013).

O objetivo deste trabalho foi apresentar uma revisão da literatura sobre as propriedades antimicrobianas atribuídas ao kefir.

De acordo com Guerra; Pastrana (2002), bactérias ácido-lácticas podem crescer em meios de cultura alternativos sem perder a habilidade de produzir compostos antimicrobianos. O interesse em tais espécies aumentou nos últimos anos, viabilizando o uso desses micro-organismos probióticos como conservantes e inibidores naturais em vários produtos de origem animal, como leites, carnes e derivados. Entende-se por probióticos micro-organismos vivos capazes de melhorar o equilíbrio microbiano intestinal, produzindo efeitos benéficos à saúde do indivíduo (Vasiljevic; SHAH, 2008). Embora ainda não esteja bem esclarecido, três possíveis mecanismos de ação são atribuídos aos probióticos:

1. a competição por nutrientes e por sítios de adesão, denominada exclusão competitiva (Cross, 2002),

2. a alteração do metabolismo microbiano, por meio do aumento ou da diminuiçáo da atividade enzimática (De VRese et al., 2001), e
3. o estímulo da imunidade do hospedeiro, por intermédio do aumento dos níveis de anticorpos e do aumento da atividade dos macrófagos (Coppola; Gil Turnes, 2004). Para ser considerado probiótico, o micro-organismo precisa ser resistente ao suco gástrico e à bile, mantendo viabilidade e atividade no intestino (SANTOS et al., 2006). A composição microbiológica do kefir o caracteriza como um alimento complexo, com um grande número de micro-organismos simbióticos, dos quais várias bactérias têm sido identificadas como probióticas (FARNwORTH et al., 2005).

A bebida kefir é obtida a partir da adição de grãos de kefir ao leite para promover a fermentaçáo. Os grãos de kefir consistem em massas gelatinosas irregulares, nas quais estão contidas bactérias ácido-lácticas, ácido-acéticas e leveduras imersas em uma matriz de proteínas e polissacarídeos (Bоsch et al., 2006). Os micro-organismos mais comumente isolados de grãos de kefir compreendem os gêneros Lactobacillus (L. brevis, L. casei, L. kefiri, L. acidophilus, L. plantarum, $L$. kefiranofaciens subsp. kefiranofaciens, $L$. kefiranofaciens subsp. kefirgranum, L. parakefir), Lactococcus (L. lactis subsp. lactis), Leuconostoc (L. mesenteroides), Acetobacter, Kluyveromyces (K. marxianus) e Saccharomyces (Takizawa et al., 1998; WiтthuhN et al., 2005; CHen et al., 2008).

Muitas espécies de Lactocbacillus são capazes de produzir uma variedade de compostos antimicrobianos, como ácidos orgânicos, dióxido de carbono, etanol, polissacarídeos e bacteriocinas, que apresentam potencial no controle de patógenos e bactérias de deterioraçáo durante a produçáo e o armazenamento dos alimentos (Messes; De VuYst, 2002). Além de conferir aroma, sabor e textura aos derivados lácteos, bactérias ácido-lácticas que apresentem açáo inibitória sobre micro-organismos indesejáveis podem ser utilizadas na indústria de alimentos como bioconservantes, em substituição aos conservantes tradicionais (Caplice; Fitzgerald, 1999).

Dias et al. (2012) avaliaram a sobrevivência de bactérias patogênicas veiculadas pelo leite durante a fermentação do kefir produzido artesanalmente. O estudo demonstrou que a fermentaçáo do leite provocada pelos micro-organismos presentes nos grãos gerou um ambiente desfavorável às bactérias testadas. Salmonella Typhimurium e Salmonella Enteritidis não foram recuperadas após 24 horas de fermentação do kefir. Os ácidos orgânicos, lático e acético, resultantes do catabolismo dos carboidratos contribuem para o decréscimo do $\mathrm{pH}$, tornando o ambiente hostil para a maioria dos micro-organismos (Bosch et al., 2006; Farnworth, 2005; Magalhâes et al., 2010). Garrote et al. (2000) atribuíram o efeito bacteriostático do kefir aos ácidos orgânicos produzidos.

CZAmanski (2003), pesquisando a ação antimicrobiana do kefir sobre micro-organismos Gram-negativos, observou inativação de Salmonella após 60 minutos de exposição. Dias (2011) estudou a atividade antimicrobiana de 60 micro-organismos 
isolados de gráos de kefir por meio do teste do antagonismo e do teste de inibiçáo com sobrenadantes contra Salmonella Enteritidis, Salmonella Typhimurium, E. coli, S. aureus e $L$. monocytogenes. $\mathrm{O} \mathrm{pH}$ dos sobrenadantes foi determinado antes de testar a atividade antimicrobiana e o teste foi repetido após o tratamento com substância neutralizante $(\mathrm{NaOH})$, a fim de excluir a ação dos ácidos orgânicos. Três (12\%) isolados apresentaram atividade antimicrobiana, demonstrando que a acidificação não é o único fator envolvido na inibição dos patógenos. L. monocytogenes não foi inibida após a neutralização do sobrenadante. GonZÁlez et al. (2007) testaram a ação inibitória de 137 cepas de Lactobacillus e também não verificaram atividade contra $L$. monocytogenes após a neutralização do $\mathrm{pH}$. Os resultados desses estudos são sugestivos de que as substâncias bioativas produzidas pelas espécies de Lactobacillus, embora apresentem ação inibitória sobre importantes micro-organismos patogênicos, não são capazes de afetar o crescimento de L. monocytogenes.

Segundo Rodrigues et al. (2005), exopolissacarídeos denominados kefiran, produzidos por alguns Lactobacillus isolados de grãos de kefir, têm sido responsabilizados pelas propriedades antimicrobianas. Em estudo comparativo da atividade antimicrobiana de kefir, extrato de kefiran e antibióticos, esses autores demonstraram que o kefiran foi capaz de inibir sete bactérias testadas. Nesse trabalho, os autores também testaram a atividade cicatrizante do kefir e do kefiran e perceberam uma melhora nas feridas cutâneas de ratos infectadas com $S$. aureus, em comparação com os tratados com a emulsão de clostebol-neomicina. Medrano et al. (2008) estudaram o efeito protetor do kefiran sobre células Caco-2 e demonstraram redução no efeito citopático causado por Bacillus cereus, quando as células eram cultivadas na presença de kefiran. Outros efeitos benéficos do kefiran, como modulação da resposta imune na mucosa intestinal (Medrano et al., 2011) e nas vias aéreas (Kwon, 2008) e ação anti-inflamatória (Furuno; NAKANishi, 2012) e antioxidante (Uchida et al., 2010), têm sido reportados.

De acordo com a bibliografia consultada, o kefir, por meio dos micro-organismos que o compóem e das substâncias bioativas por eles produzidas, constitui uma alternativa viável na busca de substitutivos para os antimicrobianos convencionais. Vários trabalhos demonstram o potencial uso de micro-organismos isolados de grãos de kefir diante de patógenos de origem alimentar, porém seus mecanismos de ação ainda são pouco conhecidos. Nesse sentido, mais estudos devem ser desenvolvidos a fim de esclarecer esses mecanismos e a segurança para o seu uso na Medicina e em alimentos.
ABRAHAM, A.G.; DE ANTONI, G.L. Characterization of kefir grains grow in cow's milk and soya milk. Journal of Dairy Research, v. 66, n. 2, p. 327-333, 1999. Disponível em: <http://journals. cambridge.org/action/displayAbstract?fromPage=online\& aid $=11539>$. Acesso em: 14 jan. 2013.

AHMED, Z.; WANG, Y.; AHMED, A.; KHAN, S.T.; NISA, M.; AHMAD, H.; AFREEN, A. Kefir and Health: A Contemporary Perspective. Critical Reviews in Food Science and Nutrition, v. 53, n. 5, p. 422-434, 2013. Disponível em: <http://www.tandfonline.com/doi/full/10 $.1080 / 10408398.2010 .540360>$. Acesso em: 15 fev. 2016.

BOSCH, A.; GOLOWCZYC, M.A.; ABRAHAM, A.G.; GARROTE, G.L.; DE ANTONI, G.L.; YANTORNO, O. Rapid discrimination of lactobacilli isolated from kefir grains by FT-IR spectroscopy. International Journal of Food Microbiology, v. 11 1, n. 3, p. 280-287, 2006. Disponível em: <http://www.sciencedirect.com/science/article/ pii/SO $168160506003114>$. Acesso em: 14 jan. 2013. doi: 10.1016/j.jjfoofmicro.2006.05.010.

BRASIL. Ministério da Agricultura, Pecuária e Abastecimento. Resolução n.05, de 13 de novembro de 2000. Regulamento Técnico de Identidade e Qualidade de Leites Fermentados. Diário Oficial da União, Brasília, 27 Nov. 2000. Seção I, p. 9-12. Disponível em: <http:// extranet.agricultura.gov.br/sislegis-consulta/consultarLegislacao. do?operacao=visualizar\&id=3285> . Acesso em 14 jan. 2013.
CAPLICE, E.; FITZGERALD, G.F. Food fermentations: role of microorganisms in food production and preservation. International Journal of Food Microbiology, v. 50, p. 131-149, 1999. Disponível em: <http://biotecmicro.altervista.org/Prof.\%20 C.\%20Mazzoni_files/MicrorgProduzeConservCibi.pdf $>$. Acesso em: 12 jan. 2013.

CHEN, H.C.; WANG, S.Y.; CHEN, M.J. Microbiological study of lactic acid bacteria in kefir grains by culture-dependent and cultureindependent methods. Food Microbiology, v. 25, p. 492-501, 2008. Disponível em: <http://www.sciencedirect.com/science/ article/pii/SO740002008000208>. Acesso em: 12 jan. 2013. doi: 10.106/j.fm.2008.01.003.

COPPOLA, M.M.; GIL TURNES, C. Probióticos e resposta imune. Ciência Rural, v. 34, n. 4, p. 1297-1303, 2004. Disponível em: <http://www.scielo.br/pdf/cr/v34n4/a56v34n4>. Acesso em: 14 jan. 2013.

CROSS, M.L. Microbes versus microbes: immune signals generated by probiotic lactobacilli and their role in protection against microbial pathogens. FEMS Immunology and Medical Microbiology, v. 34, n. 4, p. 245-253, 2002. Disponível em: <http://onlinelibrary. wiley.com/doi/10.1111/j.1574-695X.2002.tb00632.x/full>. Acesso em: 12 jan. 2013. doi: 10.1111/j.1574-695X.2002. tb00632.x. 
CZAMANSKY, R.T. Avaliação da atividade antimicrobiana de filtrados de quefir artesanal. 2003. 96f. Dissertação (Mestrado em Medicina Veterinária Preventiva) - Faculdade de Veterinária, Universidade Federal do Rio Grande do Sul, Porto Alegre, 2003.

DE VRESE, M.; STEGELMANN, A.; RICHTER, B.; FENSELAU, S.; LAUE, C.; SCHREZENMEIR, J. Probiotics-compensation for lactase insufficiency. American Journal of Clinical Nutrition, v. 73, n. 2, p. 421S-429S, 2001. Disponível em: <http://ajcn.nutrition.org/ content/73/2/42 1 s.full.pdf+html>. Acesso em: 04 fev. 2013.

DIAS, P.A. Atividade antimicrobiana de micro-organismos isolados de grãos de kefir. 201 1. 44f. Dissertação (Mestrado em Medicina Veterinária Preventiva) - Faculdade de Veterinária, Universidade Federal de Pelotas, Pelotas, 2011.

DIAS, P.A.; SILVA, D.T.; TEJADA, T.S.; LEAL, M.C.G.; CONCEIÇÃO, R.C.S.; TIMM, C.D. Survival of pathogenic microorganisms in kefir. Revista do Instituto Adolfo Lutz, v. 71, n. 1, p. 177181, 2012. Disponível em: <http://revista.ial.sp.gov.br/ index.php?option $=$ com_remository\&ltemid $=27 \&$ func $=$ fileinfo \&id=797>. Acesso em: 05 jan. 2013.

FARNWORTH, E.R. Kefir - a complex probiotic. Food Science (2) Technology Bulletin: Functional Foods, v. 2, n. 1, p. 1-17, 2005. Disponível em: <http://www.kefir.it/pdf/Kefir\%20a\%20 complex\%20probiotic.pdf>. Acesso em: 04 fev. 2013 . doi: 10.1616/1476-2137.13938.

FURUNO, T.; NAKANISHI, M. Kefiran suppresses antigen-induced mast cell activation. Biological and Pharmaceutical Bulletin, v. 35, n. 2, p. 178-183, 2012. Disponível em: <http://www.ncbi. nlm.nih.gov/pubmed/22293347>. Acesso em: 28 dez. 2012.

GARROTE, G.L.; ABRAHAM, A.G.; DE ANTONI, G.L. Inhibitory power of kefir: the role of organic acids. Journal of Food Protection, v. 63, n. 3, p. 364-369, 2000. Disponível em: <http://www.ingentaconnect. com/content/iafp/jfp/2000/00000063/00000003/ artO0012>. Acesso em: 28 dez. 2012.

GARROTE, G.L.; ABRAHAM, A.G.; DE ANTONI, G.L. Chemical and microbiological characterisation of kefir grains. Journal of Dairy Research, v. 68, p. 639-652, 2001. Disponível em: <http:// www.radioprotection.org/action/displayAbstract?fromPage $=$ on line\&aid $=100585>$. Acesso em: 28 dez. 2012. doi: 10.1017/ S0022029901005210.

GIBSON, G.R.; ROBERFROID, M.B. Dietary modulation of the human colonic microbiota: Introducing the concept of prebiotics. Journal of Nutrition, v. 125, n. 6, p. 1401-1412, 1995. Disponível em: <http://jn.nutrition.org/content/125/6/1401.full.pdf>. Acesso em: 28 dez. 2012.

GONZÁLEZ, L.; SANDOVAL, H.; SACRISTÁN, N.; CASTRO, J.M.; FRESNO, J.M.; TORNADIJO, M.E. Identification of lactic acid bacteria isolated from Genestoso cheese throughout ripening and study of their antimicrobial activity. Food Control, v. 18, n. 6, p. 716-722, 2007. Disponível em: <http://cat.inist.fr/?aModele=afficheN\&cpsidt=18463999>. Acesso em: 04 fev. 2013.

GUERRA, N.P.; PASTRANA, L. Nisin and pediocin production on mussel-processing waste supplemented with glucose and five nitrogen sources. Letter in Applied Microbiology, v. 34, p.
1 14-1 18, 2002. Disponível em: <http://onlinelibrary.wiley. com/doi/10.1046/j.1472-765x.2002.01054.x/full>. Acesso em: 03 fev. 2013. doi: 10.1046/j.1472-765x.2002.01054.x.

KAUR, N.; GUPTA, A.K. Applications of inulin and oligofructose in health and nutrition. Journal of Biosciences, v. 27, n. 7, p. 703-714, 2002. Disponível em: <http://link.springer.com/article/10.1007/ BF02708379\#page-1 >. Acesso em: 03 fev. 2013.

KWON, O.K.; AHN, K.S.; LEE, M.Y.; KIM, S.Y.; PARK, B.Y.; KIM, M.K.; LEE, I.Y.; OH, S.R.; LEE, H.K. Inhibitory effect of kefiran on ovalbumin-induced lung inflammation in a murine model of asthma. Archives of Pharmacal Research, v. 31, n. 12, p. 1590-1596, 2008. Disponível em: <http://link.springer.com/article/10.1007/ s12272-001-2156-4\#page-1 >. Acesso em: 05 fev. 2013. doi: $10.1007 / \mathrm{s} 12272-001-2156-4$.

MAGALHÃES, K.T.; PEREIRA, G.V.M.; DIAS, D.R.; SCHWAN, R.F. Microbial communities and chemical changes during fermentation of sugary Brazilian kefir. World Journal of Microbiology and Biotechnology, v. 26, p. 1241-1250, 2010. Disponível em: http://link.springer.com/article/10.1007/s 1 1274-0090294-x\#page-1. Acesso em: 05 fev. 2013. doi: 10.1007/ s1 1274-009-0294-x.

MARQUINA, D.; SANTOS, A.; CORPAS, I.; MUÑOZ, J.; ZAZO, J.; PEINADO, J.M. Dietary influence of kefir on microbial activities in the mouse bowel. Letters in Applied Microbiology, v. 35, n. 2, p. 136-140, 2002. Disponível em: <http://onlinelibrary.wiley.com/doi/10.1046/j.1472765X.2002.01155.x/full>. Acesso em: 05 fev. 2013. doi: 10.1046/j.1472-765X.2002.01155.x.

MEDRANO, M.; PÉREZ, P.F.; ABRAHAM, A.G. Kefiran antagonizes cytopathic effects of Bacillus Cereus extracellular factors. International Journal of Food Microbiology, v. 122, n. 1-2, p. 1-7, 2008. Disponível em: <http://www.sciencedirect.com/ science/article/pii/SO $168160507006162>$. Acesso em: 05 fev. 2013. doi: 10.1016/ifoodmicro.2007.11.046.

MEDRANO, M.; RACEDO, S.M.; ROLNY, I.S.; ABRAHAM, A.G; PÉREZ, P.F. Oral administration of kefiran induces changes in the balance of immune cells in a murine model. Journal of Agricultural and Food Chemistry, v. 59, n. 10, p. 5299-5304, 2011 . Disponível em: <http://www.researchgate.net/publication/51061856_Oral_ administration_of_kefiran_induces_changes_in_the_balance_of_ immune_cells_in_a_murine_model $>$. Acesso em: $05 \mathrm{fev} .2013$. doi: $10.1021 /$ jf 1049968.

MESSES, W.; DE VUYST, L. Inhibitory substances produced by lactobacilli isolated from sourdoughs - a review. International Journal of Food Microbiology, v. 72, p. 31-43, 2002. Disponível em: <http://www.sciencedirect.com/science/article/pii/ SO $168160501006110>$. Acesso em: 05 fev. 2013. doi: 10.1016/SO168-1605(01)00611-0.

ORDÓÑEZ, J.A. Tecnologia de Alimentos Vol. 2. Alimentos de Origem Animal. Porto Alegre: Ed. Artmed, 2005. 279 p.

PELAEZ, A.M.L. Estudio de la capacidad de los microorganismos del kefir, para inhibir el desarrollo fúngico y para secuestrar micotoxinas. 2013. 260f. Tesis Doctoral (Ciencias Biológicas) - Faculdad de Ciencias Exactas, Universidad Nacional de La Plata, La Plata, 2013. 
RODRIGUES, K.L; CAPUTO, L.R.G.; CARVALHO, J.C.T.; EVANGELISTA, J.; SCHNEEDORF, J.M. Antimicrobial and healing activity of kefir and kefiran extract. International Journal of Antimicrobiol Agents, v. 25, p. 404-408, 2005. Disponível em: <http://www.sciencedirect. com/science/article/pii/S0924857905000543>. Acesso em: 05 fev. 2013. doi: 10.1016/j.ijantimicag.2004.09.020.

SANTOS, M. S.; FERREIRA C. L. L. F.; GOMES, P. C.; SANTOS, J. L. POZZA, P. C.; TESHIMA, E. Influência do fornecimento de probiótico à base de Lactobacillus sp. sobre a microbiota intestinal de leitões. Ciência Agrotecnológica, v. 27, n. 6, p. 1395-1400. Disponível em: <http://www.scielo.br/scielo.php?script=sci_art text\&pid=S1413-70542003000600026>. Acesso em 23 maio 2016.

TAKIZAWA, S.; KOJIMA, S.; TAMURA, S.; FUJINAGASA, S.; BENNO, Y.; NAKASE, T. The composition of the Lactobacillus flora in kefir grains. Systematic and Applied Microbiology, v. 21, n. 1, p. 121 127, 1998. Disponível em: <http://www.sciencedirect.com/ science/article/pii/SO723202098800155>. Acesso em: 12 jan. 2013. doi: 10.1016/S0723-2020(98)80015-5.

UCHIDA, M.; ISHII, I.; INOUE, C.; AKISATO, Y.; WATANABE, K.; HOSOYAMA, S.; TOIDA, T.; ARIYOSHI, N.; KITADA, M.
Kefiran reduces atherosclerosis in rabbits fed a high cholesterol diet. Journal of Atherosclerosis and Thrombosis, v. 17, n. 9, p. 980-988, 2010. Disponível em: <http://www.ncbi.nlm.nih.gov/ pubmed/20543518>. Acesso em: 12 jan. 2013.

VASILJEVIC, T.; SHAH, N.P. Probiotics - From Metchnikoff to bioactives. International Dairy Journal, v. 18, n. 7, p. 714-728, 2008. Disponível em: <http://www.sciencedirect.com/science/article/ pii/S0958694608000447>. Acesso em: 12 jan. 2013. doi: 10.1016/j.idairij.2008.03.004.

VINDEROLA, C.G.; DUARTE, J.; THANGAVEL, D.; PERDIGÓN, G.;FARNWORTH, E.; MATAR, C. Immunomodulating capacity of kefir. Journal of Dairy Research, v. 72, p. 195-202, 2005. Disponível em: <http://xa.yimg.com/kq/groups/1920818/126123070/ name/jurnal+2.pdf>. Acesso em: 12 jan. 2013. doi: 10.1017/ S0022029905000828.

WITTHUHN, R.C.; SCHOEMAN, T.; BRITZ, T.J. Characterization of the microbial population at different stages of kefir production and kefir grain mass cultivation. International Dairy Journal, v. 15, p. 383-389, 2005. Disponível em: <http://www.sciencedirect.com/science/ article/pii/S0958694604002134>. Acesso em: 05 fev. 2013. doi: $10.1016 /$ j.idairyj.2004.07.016. 